\title{
Higgs physics and cosmology: gravitational waves from the electroweak phase transition
}

\author{
David Weir ${ }^{* \dagger}$ \\ Department of Physics and Helsinki Institute of Physics \\ P.O. Box 64 (Gustaf Hällströmin katu 2) \\ 00014 University of Helsinki, Finland \\ E-mail: david.weirahelsinki.fi
}

\begin{abstract}
In the Standard Model, the electroweak phase transition is a crossover. In many extensions, including those with additional charged Higgs bosons, the phase transition can be of first ordereven strongly so. In such a phase transition, the resulting collisions of bubbles of the new Higgs phase, and the associated interactions of sound waves in the plasma, are substantial sources of gravitational waves. For a phase transition at or around the electroweak scale, these gravitational waves may be detectable by future or planned missions, such as LISA. This can indirectly provide a complementary probe of particle physics beyond the Standard Model. I will review the physics that makes this possible.
\end{abstract}

Prospects for Charged Higgs Discovery at Colliders - CHARGED2018

25-28 September 2018

Uppsala, Sweden

\footnotetext{
* Speaker.

${ }^{\dagger}$ This talk presents results obtained in collaboration with Mark Hindmarsh, Stephan J. Huber, Lauri Niemi, Hiren Patel, Michael Ramsey-Musolf, Tuomas Tenkanen and Kari Rummukainen.
} 


\section{Introduction}

Over the next twenty years, gravitational waves will become a major focus not only for astrophysics but also for cosmology. The LIGO and VIRGO detectors have now seen eleven gravitational wave events [1], ten of which were black hole mergers, with one neutron star merger [2].

For cosmologists, the promise of gravitational waves lies in the fact that they allow us to see back to periods in the history of the universe when it was optically opaque (see e.g. Refs. [3, 4] for recent reviews). For example, a broad-spectrum stochastic background of gravitational waves is generically produced during inflation. This might be detectable directly by current or future interferometric experiments and missions; it is also expected to imprint itself on the B-modes of the cosmic microwave background. Other processes, such as preheating, or the evolution of a network of topological defects, can also source gravitational waves.

The LISA mission, due to launch in 2034, will consist of three satellites in a triangular configuration, with two laser links between each spacecraft [5]. It will be able to detect gravitational waves from a wide range of astrophysical sources, such as galactic binaries (including white dwarfs, neutron stars, and stellar-origin black holes), massive black hole mergers, and the inspiral of stellar origin black holes into much larger massive black holes.

However, LISA is - fortuitously - also configured in a way that will allow us to see gravitational waves from a wide variety of phase transition scenarios at or around the electroweak scale (see e.g. Refs. [6, 7]). Indeed, detecting (or ruling out) such a scenario is part of the LISA science case. Unlike gravitational waves from inflation, however, the signal is strongly peaked. This peak, including its amplitude, depends fairly strongly on the details of the phase transition.

In the sections that follow, we will see how the electroweak phase transition is able to produce a stochastic background of gravitational waves.

\section{The electroweak phase transition}

The electroweak phase transition in the Standard Model is known to be a crossover for the physical Higgs mass of $125 \mathrm{GeV}$. This was established over twenty years ago through lattice simulations of a dimensionally-reduced model that accurately, but efficiently, describes the longdistance physics of the model [8,9]; it was then verified by other means [10, 11]. However, by adding additional degrees of freedom, such as extra charged scalar fields, it is possible to get a first-order phase transition during electroweak symmetry breaking. This is particularly true if the additional fields are light.

A first-order phase transition proceeds by the nucleation of scalar field bubbles of the true vacuum inside the old, false vacuum, phase. These bubbles expand and merge, filling the space with the new phase (see Fig. 1). As they expand, the walls of the bubbles interact with the plasma formed by the rest of the particles and, in almost all electroweak phase transition scenarios [13], approach a terminal wall velocity. Meanwhile, a reaction front is set up in the vicinity of the expanding bubble walls, producing an expanding shell of kinetic energy in the plasma. After the bubbles collide, these shells continue to expand at the speed of sound.

The bubble collisions, and in particular the overlapping sound waves set up in the hot Standard Model plasma, mean that a first-order electroweak phase transition would be an efficient source of 


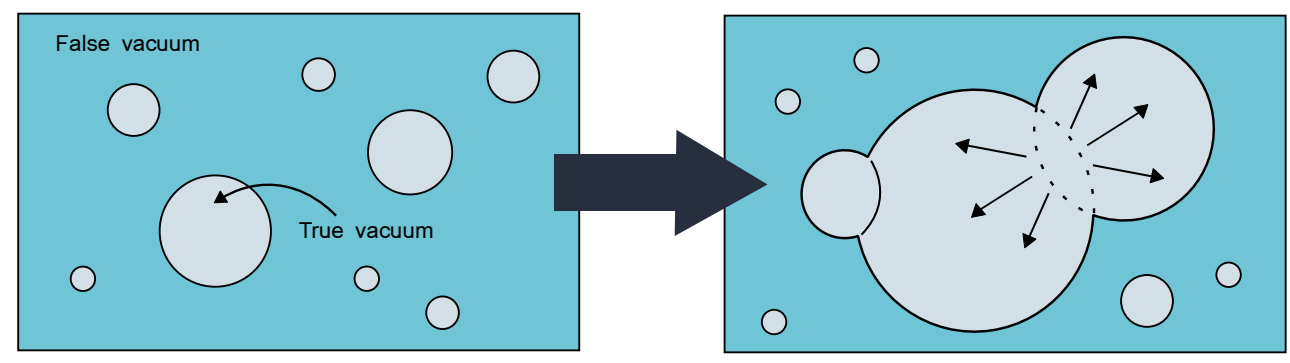

Figure 1: Schematic illustration of a first-order phase transition, after Ref. [12]. Bubbles of the true vacuum are nucleated in the false vacuum. These expand, and collide. Gravitational waves are sourced both by the bubble collisions themselves, and by the overlapping sound shells after the bubbles have merged. Credit: A. Kormu.

gravitational waves [14]. If the walls move subsonically and there is sufficient CP violation in the theory, this also represents a viable framework in which to achieve electroweak baryogenesis (see Ref. [15] for a review).

In some cases, the electroweak phase transition is not the only symmetry-breaking phase transition in the model. There may be a first-order phase transition only involving other fields, followed by the electroweak phase transition (which is usually then first order, too). Such 'two-step' scenarios have been studied in Refs. [16, 17, 18]. In fact, these papers focussed on a triplet extension of the Standard Model, which we investigated using the technique of high-temperature dimensional reduction in Ref. [19] (see Ref. [20] for a thorough discussion of dimensional reduction in the context of the electroweak phase transition).

\subsection{Dimensional reduction}

The full four-dimensional theory of the electroweak sector of the Standard Model, or one of its extensions, is difficult to study nonperturbatively. If we instead construct a three-dimensional high-temperature effective field theory, we can handle the infrared physics in a consistent way; integrate most of the field content out; and map the four-dimensional parameters onto a much simpler field theory. In the case of the gauge-Higgs minimal model of Refs. [8, 9], there are just two free parameters: the quadratic and quartic terms in the three-dimensional scalar potential. Higher dimensional operators are neglected, and all other fields have been integrated out.

Equipped with a dimensionally reduced model, we can use the three-dimensional quantum field theory to study the phase transition in the original model. As the dimensionally reduced theory correctly describes the infrared physics of the full finite-temperature field theory, we can use it to study the phase diagram and determine quantities such as the latent heat and critical temperature. One can use the three-dimensional effective potential [21], or simulate the theory on the lattice [8]. The lattice simulations of the three-dimensional theory were used to show that the endpoint of the first-order critical line lies around $80 \mathrm{GeV}$.

\subsection{Results for extended models}

If the additional degrees of freedom beyond the Standard Model Higgs are sufficiently heavy, 


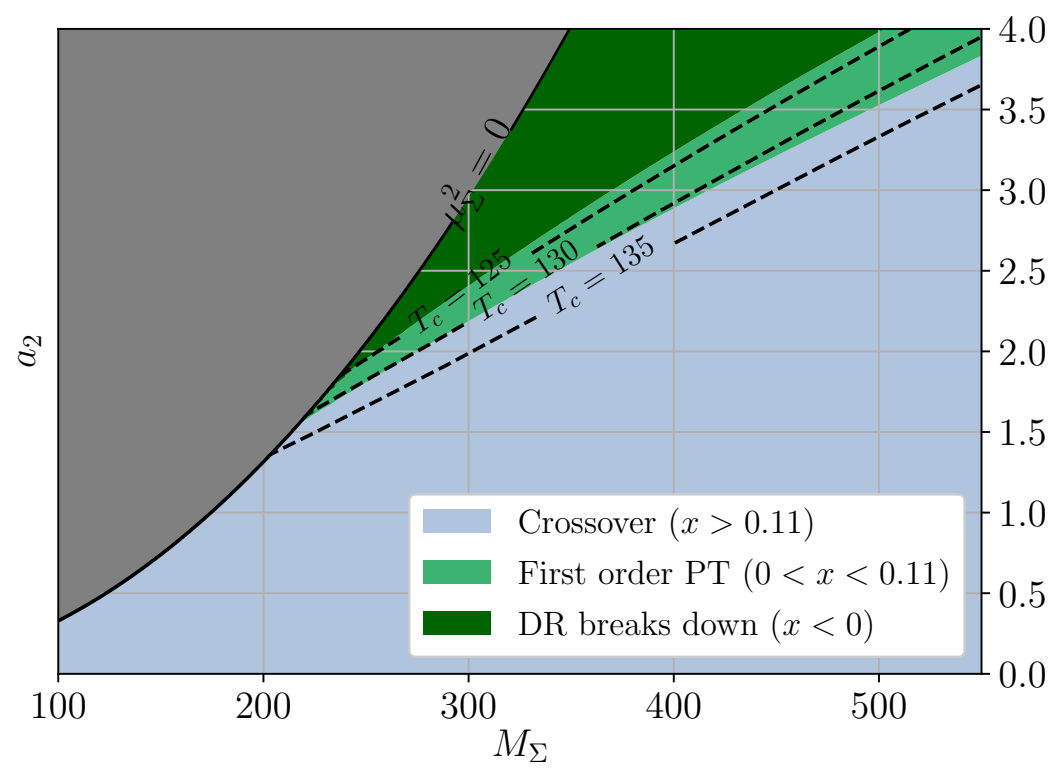

Figure 2: Figure from Ref. [19] showing the regions of parameter space for which the existing nonperturbative results for the phase transition in the three-dimensional Standard Model effective field theory can be applied to the real triplet extension of the Standard Model. The axes are the triplet mass $M_{\Sigma}$ and the portal coupling $a_{2}$. The dashed contours give the critical temperature in $\mathrm{GeV}$. The light blue region is where the electroweak phase transition is a crossover in this model; in the light green region the phase transition is of first-order. The dark green region shows where the dimensional reduction fails to work - principally due to neglected higher-dimensional operators, while in the grey region the real triplet is light and must be included in the dimensional reduction.

then they can be integrated out during the dimensional reduction. The logical conclusion of this approach is to integrate out all the additional degrees of freedom, leaving the same three-dimensional theory as describes the minimal Standard Model electroweak sector, albeit with different matching relations between the three- and four-dimensional theories. This allows us to apply the results of Ref. [8] to this new theory to determine the regions of parameter space for which a first-order electroweak phase transition occurs (this type of mapping was first suggested in Ref. [22]).

In Ref. [19], we used this approach to study a theory with an additional real triplet field. The result of this reuse of existing results is shown in Fig. 2. While attractive, this technique is not without problems or limitations. If the additional field content - in our example, the triplet field is light, then it cannot be integrated out and the existing results cannot be used. Even in cases where the field is sufficiently heavy to be integrated out, the dimensional reduction may neglect higherdimensional operators which ought to have been included. As the largest source of systematic error in such a calculation, it is important to verify the importance of higher-dimensional operators.

For cases where the mapping onto the minimal three-dimensional Standard Model does not work, additional simulations will be required to reliably study the theory nonperturbatively. On the other hand, in cases where it does work the critical temperature, latent heat and other quantities needed for computing the gravitational wave power spectrum can be obtained from the literature. 


\section{Gravitational waves}

The previous section summarised some of the ways in which a first-order electroweak phase transition can be produced by the addition of extra physics, for example by the presence of additional charged Higgs fields, and discussed ways of computing the phase diagram and relevant parameters for such scenarios. In this section we turn our attention to the gravitational wave power spectrum that may be observed in such a situation.

It has generally been thought that there are three sources of gravitational waves in a thermal first-order phase transition: the collision of the scalar field bubble walls, the overlapping sound shells formed by the reaction fronts after the bubbles have collided, and turbulence formed in the plasma long after the bubbles have merged.

As the initial bubble collisions are short-lived and only involve the gradient energy of the scalar field bubble walls, only in cases where the walls accelerate to ultrarelativistic speeds is it likely that bubble wall collisions are an important source of gravitational waves; we do not consider such socalled 'runaway' scenarios any further as they do not seem likely [13]. On the other hand, the exact amplitude of the turbulent power spectrum, as well as how quickly it develops, remains unclear and is worthy of further investigation.

\subsection{Simulations}

For thermal phase transitions at the electroweak scale, then, the overlapping sound waves are likely to represent the most significant source of gravitational waves. Their spectral shape and properties have been extensively studied in simulations over the past few years [14, 23], and indeed an analytical model has been developed [24].

The spectral shape proposed in Ref. [6] was developed as a result of simulations, and further validated in Ref. [24]. These simulations employ a system of a relativistic scalar field $\phi$, which models the scalar field driving the phase transition (e.g. the Higgs field), and a relativistic ideal fluid $u^{\mu}$, which models the Standard Model plasma. A phenomenological damping term is introduced which converts scalar field gradients into fluid kinetic energy. This model was widely used in simple spherically-symmetric simulations of bubble nucleation for first-order phase transitions twenty-five years ago (see e.g. Ref. [25]). It was first studied in three dimensions - and applied to gravitational wave production from first-order thermal phase transitions - in Ref. [14].

These simulations have some limitations. One issue is that the dynamic range available in state-of-the-art three-dimensional computer simulations allows at most three orders of magnitude of length scales to be resolved; this is in contrast to the situation in the early universe, where many orders of magnitude separated the (microscopic) bubble wall width from the (cosmological) bubble radius. However, with care, robust results can be extrapolated.

Another issue is that we are unable to see the finite duration of the sound source in our simulations so far. There is a time scale $\tau_{\mathrm{sh}}=H_{*} R_{*} / \bar{U}_{\mathrm{f}}$, where $H_{*}$ is the Hubble constant at the time of gravitational wave production, $R_{*}$ is the average bubble radius, and $\bar{U}_{\mathrm{f}}$ is the average plasma four-velocity. For many parameter choices [26], this timescale is less than the Hubble time, and so the sound source may be attenuated by processes other than the expansion of the universe (in the plots in this proceedings we take this cutoff time into account). Work to investigate the fate of the sound source - and whether turbulence forms - is underway. 


\subsection{PTPlot.org: an online tool}

To streamline the calculation of the gravitational wave power spectrum for electroweak-scale first-order phase transitions, we have developed an online tool [27], PTPlot, that will allow the computation of predicted gravitational wave power spectra for first-order phase transition scenarios relevant for LISA (see Fig. 3 for an example).

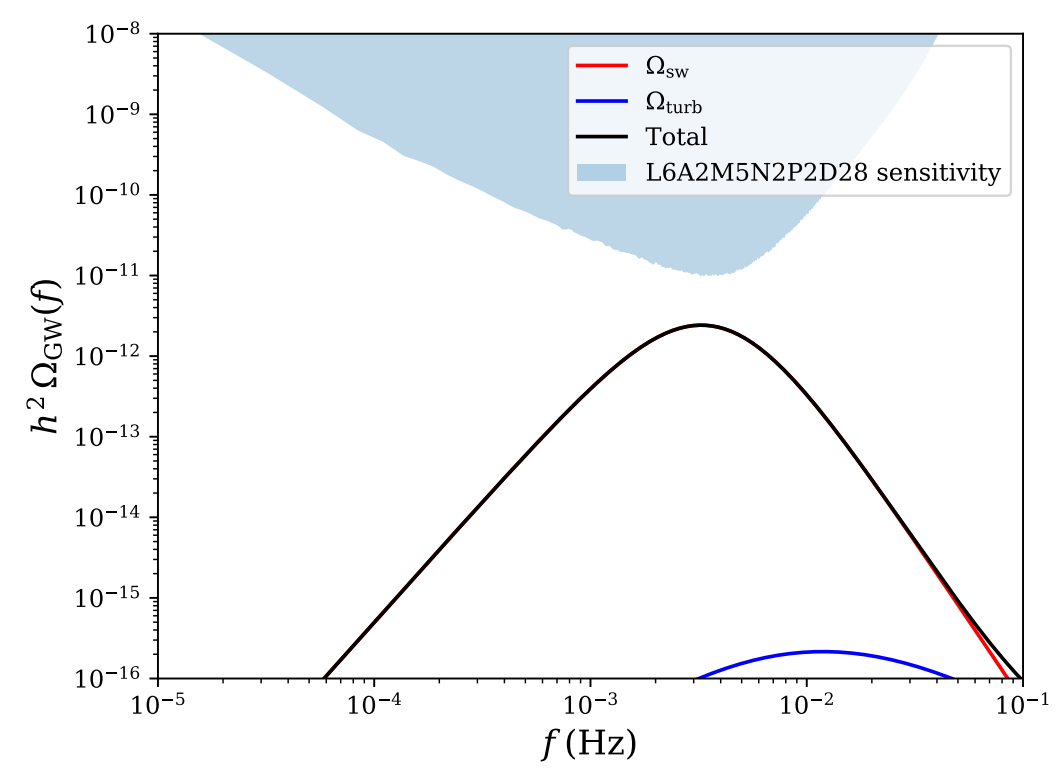

Figure 3: Typical gravitational wave power spectrum for a first-order phase transition that may be observable at LISA. This point, with $T_{*}=51.64 \mathrm{GeV}, \alpha=0.111$ and $\beta / H_{*}=663$ and $v_{\mathrm{W}}=0.95$, corresponds to the ' $\mathrm{C}$ ' benchmark point for the two Higgs doublet model (2HDM) in Ref. [6], Table 3. Note that a conservative limit on the sound source lifetime has been applied, as discussed in the text. The SNR from the sound wave source alone is about 150 in this case (with a five year mission duration). Note that the sensitivity curve used here and in subsequent figures will be supplanted by that in the LISA Science Requirements Document.

It also computes the signal-to-noise ratio (SNR) and can create parametric SNR plots, as well. For these purposes, the gravitational wave power is taken to be sourced entirely by sound waves [23], but with attenuation due to the conservative assumption that the sound wave source will not last longer than one fluid turnover time [26]. Examples of such plots are shown in Fig. 4.

\section{Outlook}

The direct detection of gravitational waves by the LIGO and VIGRO collaborations has catalysed interest in both astrophysical and cosmological sources of gravitational waves. At the same time, the LISA mission is now in preparation and due to launch in 2034.

The design of LISA is such that it will be able to detect gravitational waves from strong firstorder phase transitions at the electroweak scale. Indeed, it can be seen as a sort of particle physics experiment, with the ability to probe some models and regions of parameter space better than current or future colliders (see e.g. Ref. [28]). 

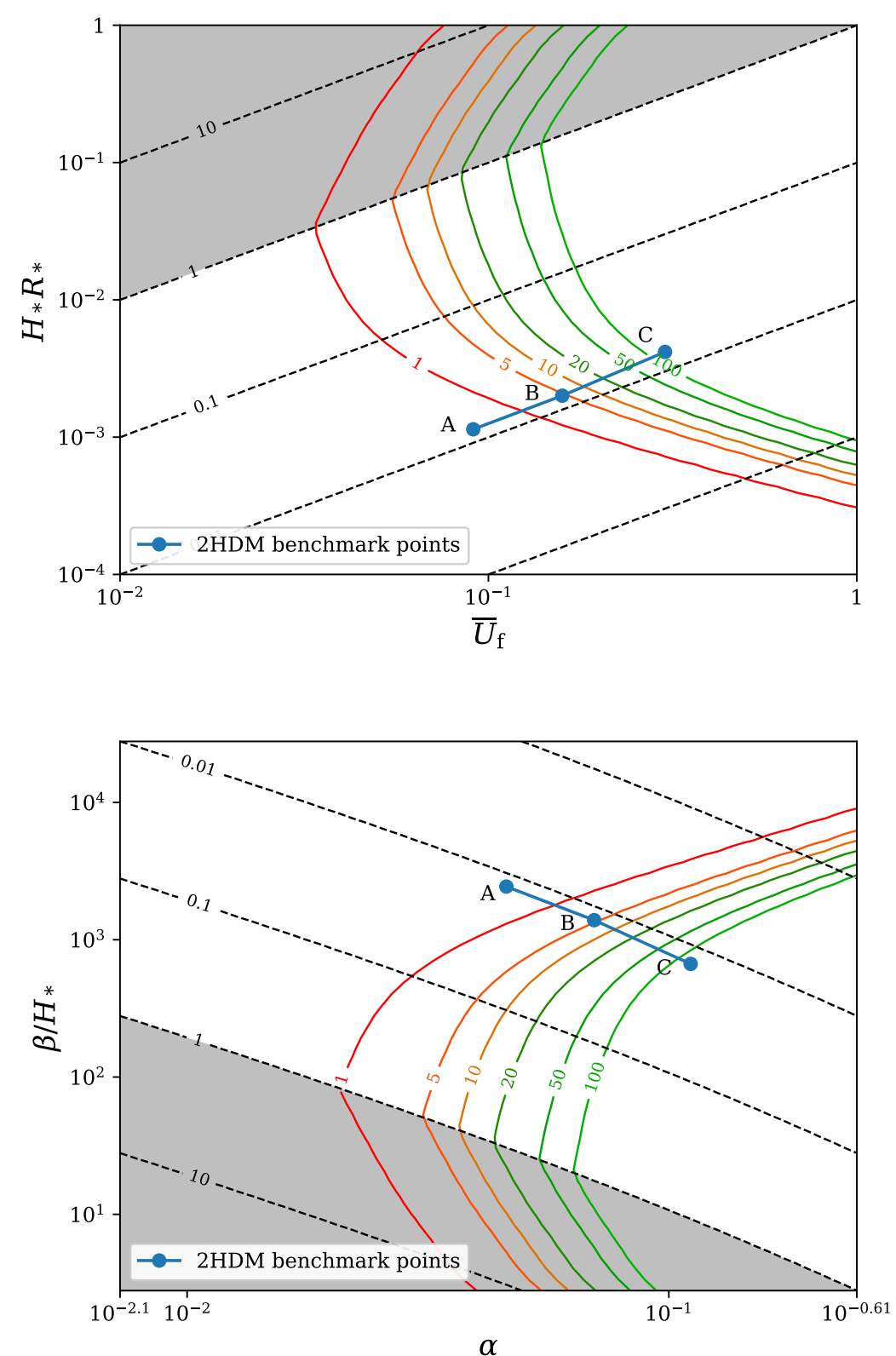

Figure 4: Contour plots of the SNR for the 2HDM benchmark points in Ref. [6], Table 4. $T_{*}$ is taken to be $50 \mathrm{GeV}$, and the plots can be compared with the top-right pane of Ref. [6], Fig. 4. At top, the SNR contours are plotted in terms of the effective average fluid velocity $\bar{U}_{\mathrm{f}}$ and the bubble radius as a fraction of the Hubble radius $\left(H_{n} R_{*}\right)$. At bottom the contours are plotted directly in terms of the strength and inverse phase transition duration parameters $\alpha$ and $\beta / H_{*}$, respectively. The dashed lines show the shock formation time $\tau_{\mathrm{sh}}=H_{*} R_{*} / \bar{U}_{\mathrm{f}}$; the grey shaded region shows where this is longer than a Hubble time and the sound wave results are expected to be most reliable [26]. In the unshaded region, we assume the source turns off after $\tau_{\mathrm{sh}}$ and the SNR contours take this into account. 
Other future missions, such as the Einstein Telescope or DECIGO, will further grow the range of early-universe phenomena and energy scales that can be probed with gravitational waves.

Before LISA launches in 2034, however, much remains to be done. The quantities that determine the gravitational wave power spectrum, such as the critical and nucleation temperatures, latent heat, phase transition duration, and bubble wall velocity, ought to be computed with greater precision in models of interest. The gravitational wave power spectrum from such a scenario must be better studied, to establish firmly the lifetime and fate of the sound wave source of gravitational waves; the development of turbulence must also be investigated. Work on all these exciting topics is already underway.

\section{Acknowledgments}

In addition to the fruitful and long-lasting support and collaboration of all my collaborators on the work described here, I am grateful to Anna Kormu for supplying Fig. 1. I acknowledge support from Academy of Finland, grant number 286769, and the Research Funds of the University of Helsinki.

\section{References}

[1] B. P. Abbott et al. [LIGO Scientific and Virgo Collaborations], GWTC-1: A Gravitational-Wave Transient Catalog of Compact Binary Mergers Observed by LIGO and Virgo during the First and Second Observing Runs, arXiv:1811.12907 [astro-ph.HE].

[2] B. P. Abbott et al. [LIGO Scientific and Virgo Collaborations], GW170817: Observation of Gravitational Waves from a Binary Neutron Star Inspiral, Phys. Rev. Lett. 119 (2017) no.16, 161101 [arXiv:1710.05832 [gr-qc]].

[3] C. Caprini and D. G. Figueroa, Cosmological Backgrounds of Gravitational Waves, Class. Quant. Grav. 35 (2018) no.16, 163001 [arXiv:1801.04268 [astro-ph.CO]].

[4] N. Christensen, Stochastic Gravitational Wave Backgrounds, Rept. Prog. Phys. 82 (2019) no.1, 016903 [arXiv:1811.08797 [gr-qc]].

[5] H. Audley et al. [LISA Collaboration], Laser Interferometer Space Antenna, arXiv:1702.00786 [astro-ph.IM].

[6] C. Caprini et al., Science with the space-based interferometer eLISA. II: Gravitational waves from cosmological phase transitions, JCAP 1604 (2016) no.04, 001 [arXiv:1512.06239 [astro-ph.CO]].

[7] D. J. Weir, Gravitational waves from a first order electroweak phase transition: a brief review, Phil. Trans. Roy. Soc. Lond. A 376 (2018) no.2114, 20170126 [arXiv:1705.01783 [hep-ph]].

[8] K. Kajantie, M. Laine, K. Rummukainen and M. E. Shaposhnikov, The Electroweak phase transition: A Nonperturbative analysis, Nucl. Phys. B 466 (1996) 189 [hep-lat/9510020].

[9] K. Kajantie, M. Laine, K. Rummukainen and M. E. Shaposhnikov, Is there a hot electroweak phase transition at $m(H)$ larger or equal to $m(W)$ ?, Phys. Rev. Lett. 77 (1996) 2887 [hep-ph/9605288].

[10] M. Gurtler, E. M. Ilgenfritz and A. Schiller, Where the electroweak phase transition ends, Phys. Rev. D 56 (1997) 3888 [hep-lat/9704013]. 
[11] F. Csikor, Z. Fodor and J. Heitger, Endpoint of the hot electroweak phase transition, Phys. Rev. Lett. 82 (1999) 21 [hep-ph/9809291].

[12] Phase Transitions in the Early Universe, http://www.damtp.cam.ac.uk/research/gr/public/cs_phase.html, accessed 13.12.2018.

[13] D. Bodeker and G. D. Moore, Electroweak Bubble Wall Speed Limit, JCAP 1705 (2017) no.05, 025 [arXiv:1703.08215 [hep-ph]].

[14] M. Hindmarsh, S. J. Huber, K. Rummukainen and D. J. Weir, Gravitational waves from the sound of a first order phase transition, Phys. Rev. Lett. 112 (2014) 041301 [arXiv:1304.2433 [hep-ph]].

[15] D. E. Morrissey and M. J. Ramsey-Musolf, Electroweak baryogenesis, New J. Phys. 14 (2012) 125003 [arXiv:1206.2942 [hep-ph]].

[16] H. H. Patel and M. J. Ramsey-Musolf, Stepping Into Electroweak Symmetry Breaking: Phase Transitions and Higgs Phenomenology, Phys. Rev. D 88 (2013) 035013 [arXiv:1212.5652 [hep-ph]].

[17] N. Blinov, J. Kozaczuk, D. E. Morrissey and C. Tamarit, Electroweak Baryogenesis from Exotic Electroweak Symmetry Breaking, Phys. Rev. D 92 (2015) no.3, 035012 [arXiv:1504.05195 [hep-ph]].

[18] S. Inoue, G. Ovanesyan and M. J. Ramsey-Musolf, Two-Step Electroweak Baryogenesis, Phys. Rev. D 93 (2016) 015013 [arXiv:1508.05404 [hep-ph]].

[19] L. Niemi, H. H. Patel, M. J. Ramsey-Musolf, T. V. I. Tenkanen and D. J. Weir, Electroweak phase transition in the $\Sigma S M$ - I: Dimensional reduction, arXiv:1802.10500 [hep-ph].

[20] K. Kajantie, M. Laine, K. Rummukainen and M. E. Shaposhnikov, Generic rules for high temperature dimensional reduction and their application to the standard model, Nucl. Phys. B 458 (1996) 90 [hep-ph/9508379].

[21] M. Laine, The Two loop effective potential of the 3-d SU(2) Higgs model in a general covariant gauge, Phys. Lett. B 335 (1994) 173 [hep-ph/9406268].

[22] J. M. Cline and K. Kainulainen, Supersymmetric electroweak phase transition: Beyond perturbation theory, Nucl. Phys. B 482 (1996) 73 [hep-ph/9605235].

[23] M. Hindmarsh, S. J. Huber, K. Rummukainen and D. J. Weir, Shape of the acoustic gravitational wave power spectrum from a first order phase transition, Phys. Rev. D 96 (2017) no.10, 103520 [arXiv:1704.05871 [astro-ph.CO]].

[24] M. Hindmarsh, Sound shell model for acoustic gravitational wave production at a first-order phase transition in the early Universe, Phys. Rev. Lett. 120 (2018) no.7, 071301 [arXiv:1608.04735 [astro-ph.CO]].

[25] J. Ignatius, K. Kajantie, H. Kurki-Suonio and M. Laine, The growth of bubbles in cosmological phase transitions, Phys. Rev. D 49 (1994) 3854 [astro-ph/9309059].

[26] J. Ellis, M. Lewicki and J. M. No, On the Maximal Strength of a First-Order Electroweak Phase Transition and its Gravitational Wave Signal, [arXiv:1809.08242 [hep-ph]].

[27] PTPlot, http://www.ptplot.org, accessed 14.1.2019.

[28] M. Chala, C. Krause and G. Nardini, Signals of the electroweak phase transition at colliders and gravitational wave observatories, JHEP 1807 (2018) 062 [arXiv:1802.02168 [hep-ph]]. 